\title{
Alterations in function and expression of $A B C$ transporters at blood-brain barrier under diabetes and the clinical significances
}

\section{Li Liu and Xiao-Dong Liu *}

Center of Drug Metabolism and Pharmacokinetics, College of Pharmacy, China Pharmaceutical University, Nanjing, China

Edited by:

George Tegos, University of New

Mexico, USA

\section{Reviewed by:}

Adriana Maggi, University of

Milan, Italy

Filippo Caraci, University of

Catania, Italy

\section{*Correspondence:}

Xiao-Dong Liu, Center of Drug Metabolism and Pharmacokinetics, China Pharmaceutical University,

24 Tongjia Lane, Nanjing 210009,

Jiangsu, China

e-mail:xdliu@cpu.edu.cn
Diabetes is a systematic metabolic disease, which often develops a number of well-recognized vascular complications including brain complications which may partly result from the dysfunction of blood-brain barrier (BBB). BBB is generally considered as a mechanism for protecting the brain from unwanted actions resulting from substances in the blood and maintaining brain homeostasis via monitoring the entry or efflux of compounds. ATP-binding cassette (ABC) family of transporters including P-glycoprotein (P-GP) and breast cancer-related protein (BCRP), widely expressed in the luminal membrane of the microvessel endothelium and in the apical membrane of the choroids plexus epithelium, play important roles in the function of BBB. However, these transporters are easily altered by some diseases. The present article was focused on the alteration in expression and function of both P-GP and BCRP at BBB by diabetes and the clinical significances.

Keywords: blood-brain barrier, $A B C$ transporters, diabetes, Alzheimer's disease, amyloid $\beta$-peptide, ABCB1, ABCG2

\section{INTRODUCTION}

The blood-brain barrier (BBB) is a multicellular vascular structure that separates the central nervous system (CNS) from the peripheral blood circulation. The basic structural and functional unit of the BBB is considered to be the so-called neurovascular unit, which is composed of at least four cell types: brain capillary endothelial cells comprise the brain capillaries of the cerebral vasculature; pericytes sit on top of endothelial cells sharing the same basal membrane; astrocytes surround the brain capillaries and cover them with their endfeet; and neurons directly innervate the microvasculature. Together these cells of the neurovascular unit control barrier function. By tightly controlling the passage of molecules and ions, instantaneously delivering nutrients and oxygen according to current neuronal needs and protecting the brain from toxins and pathogens, the BBB maintains an environment that allows neurons to function properly (Obermeier et al., 2013).

Beyond barrier function, influx and efflux are actively regulated at the blood-brain interface. At the molecular level, several factors contribute to the unique functional role of the BBB. Tight junctions in the capillary endothelium seal the spaces between neighboring endothelial cells and represent a passive, physical barrier that restricts paracellular diffusion of solutes from blood to brain. To meet the high nutrient and energy demand of the brain, influx transporters facilitate brain uptake of glucose, amino acids, ions, and other nutrients from the blood. ATP-binding cassette (ABC) efflux transporters on the other hand extrude metabolic wastes into the blood and form a selective, active barrier protecting the CNS by limiting xenobiotics, including toxins and a large number of drugs, from entering the brain (Hartz and Bauer, 2011). Thus, the BBB is a complex and fine-tuned transport machine that balances influx of nutrients and efflux of wastes, toxins, and drugs to maintain CNS homeostasis. The ABC efflux proteins of this transport machinery are critical for the functional barrier and present a formidable impediment to brain delivery of therapeutic drugs.

$\mathrm{ABC}$ transporters belong to one of the largest and most ancient protein superfamilies and are highly conserved between species and throughout evolution. $\mathrm{ABC}$ transporters are transmembrane proteins that transport lipids, sterols, metabolic wastes, and therapeutic drugs across intra- and extracellular membranes. These transport processes are ATP-driven (active transport) and can, therefore, be directed against a solute's concentration gradient. By now, the $A B C$ transporter superfamily comprises 49 human proteins divided into seven subfamilies that have been designated ABCA to ABCG (Pahnke et al., 2014). They are expressed in every cell type of the brain and mediate the transport of a wide variety of substances.

For vertebrates, three $\mathrm{ABC}$ subfamilies, $\mathrm{B}, \mathrm{C}$, and $\mathrm{G}$, contain transporters that function as multispecific, ATP-driven efflux pumps, and largely handle foreign chemicals (xenobiotics). As a rule, these ABC transporters are expressed in all cells, but they are most highly expressed in barrier (such as the BBB) and excretory tissues. Certainly, for an efflux transporter to be effective in limiting blood to brain movement of drugs and neurotoxicants and driving efflux of potentially toxic metabolites, it should be localized to the luminal plasma membrane. Functional studies with wild-type and knock-out rodents as well as immunohistochemistry indicate that luminal 
membrane localization is certain for P-glycoprotein (ABCB1), Multidrug Resistance-Associated Protein 2, MRP2 (ABCC2), and breast cancer resistance protein (ABCG2) (BCRP). They have the potential to affect many signaling molecules, waste products of normal metabolism, therapeutic drugs, environmental toxicants, and toxicant metabolites efflux out of the CNS (Miller, 2014).

Under the physiological state, BBB is generally considered as a mechanism for protecting the brain from unwanted actions resulting from substances in the blood and maintaining brain homeostasis via monitoring the entry or efflux of compounds. The BBB is not a static anatomical boundary, but a dynamic interface capable of rapid response to stressors including hypoxia, inflammation, trauma, and pain. In a number of brain pathologies, loss of $\mathrm{BBB}$ function and changes in $\mathrm{ABC}$ transporter expression have been suggested as a prognostic factor in the progression of these diseases (Wanek et al., 2013). Furthermore, the $\mathrm{BBB}$ and $\mathrm{ABC}$ transporter dysfunction exacerbates numerous diseases and pathologies including stroke, Alzheimer disease, Parkinson disease, diabetes, acute liver failure, etc. Loss of BBB integrity (i.e., leak or dysfunction of $\mathrm{ABC}$ transporters) exposes the brain to potentially harmful concentrations of substances in the peripheral circulation (e.g., ions, amino acids, neurotransmitters, proteins, and other macromolecules) that may disrupt brain homeostasis and adversely affect neuronal signaling (Abbott et al., 2010).

Diabetes mellitus (DM) is a systematic metabolic disease, which develops a number of well-recognized macro- and microvascular complications related to endothelial dysfunction (Stumvoll et al., 2005). Diabetic macrovascular complications involve vessel obstructions, such as coronary artery diseases, atherosclerosis, and peripheral vascular diseases. Microvascular pathologies include retinopathy, nephropathy, and neuropathy. Direct damage to small blood vessels, particularly by hyperglycemia, is manifested by endothelial dysfunction, diminished perfusion, abnormal endothelial cell proliferation, and increased vessels permeability. Type $2 \mathrm{DM}$ patients exhibit similar microvascular damage within the CNS which may result in increased incidence of cognitive deterioration, vascular dementia, lacunar infarcts, hemorrhages and Alzheimer's disease (AD) (Serlin et al., 2011). These diabetes induced brain microvascular complications have disgusting consequences in dysfunction of the BBB. Several phenomena known to disrupt the BBB integrity, such as transient cerebral ischemia, hypertension and hyperosmolality, are commonly associated with DM. DM is also associated with changes in $\mathrm{ABC}$ transport functions in the cerebral microvessels. Under diabetic conditions, the integrity of $\mathrm{BBB}$ is compromised and molecules that are normally confined to the blood may enter the parenchyma leading to dramatic changes in brain structure and function (Alves et al., 2012). The diabetes-induced perturbations to cerebral microvessels may disrupt homeostasis and contribute to long-term cognitive and functional deficits of the CNS. The present article summarizes and highlights the accumulating evidence in the literature which describe the role of altered expression and function of both $\mathrm{P}-\mathrm{GP}$ and BCRP in the pathogenesis of diabetes and the clinical significances.

\section{ALTERATION IN BBB PERMEABILITY BY DIABETES ALTERED BLOOD-BRAIN BARRIER STRUCTURE IN DIABETES MELLITUS}

Alteration of cerebral microvessel structure, notably decreased capillary density and disruption of tight junction function, is one of the important reasons that diabetes disrupts BBB function. Although BBB permeability under diabetic status has been widely investigated, the results are often controversial. Dai et al. reported that no obvious differences in the staining pattern of IgG and albumin were observed between brain samples of persons with diabetes and controls (Dai et al., 2002). But Starr's study showed the increased BBB permeability to gadolinium diethylenetriamine pentaacetic acid in patients with type 2 diabetes using gadolinium magnetic resonance imaging (Starr et al., 2003). Antibodies against serum S100B and NSE (CNS proteins) were found to be significantly increased in both type 1 and type 2 diabetic subjects compared to controls, implying that diabetes in humans may be associated with alterations in the integrity of the BBB (Hovsepyan et al., 2004). Animal experiment demonstrated that diabetes significantly reduced cerebral tight junction protein occludin content, but another tight junction protein zonula occludens-one content was not significantly altered, indicating that diabetes alters the molecular anatomy of the tight junctions in cerebral tissue by altering the content of select structural proteins (Chehade et al., 2002).

\section{METABOLIC CHANGES ON BBB IN DIABETES MELLITUS}

Several studies have exhibited alterations in some nutrient transporters at $\mathrm{BBB}$ under diabetic status. In hyperglycemic non-obese diabetic mice, diabetes down-regulated both BBB permeability to glucose and transporter maximal velocity of glucose without alteration in the half-saturation constant (Cornford et al., 1995), which may be attributed to the downregulated glucose transporters (Hou et al., 2007). The decrease in expression of glucose transporters at $\mathrm{BBB}$ impairs glucose transport into the brain, leading to mental retardation (De Vivo et al., 1991) although there was a report showing transport of glucose across BBB was not altered in poorly controlled type 1 diabetes (Fanelli et al., 1998). Influx of vitamin $\mathrm{C}$ to the brain is also mediated via the glucose transporter, indicating that the decreased glucose transporter may be responsible for depleted brain content of vitamin $\mathrm{C}$ in diabetic animals (Minamizono et al., 2006). In addition, diabetes decreased monocarboxylic acid transporter expression, leading to increase in BBB permeability to acetate acid (Mason et al., 2006). Insulin uptake by brain was reported to increase under diabetic status, however, this increase was not due to acute changes in the serum levels of glucose or insulin, altered vascular space, or catabolic events (Banks et al., 1997).

\section{MECHANISMS OF BBB BREAKDOWN IN DIABETES MELLITUS}

The underlying molecular changes leading to BBB dysfunction under diabetic condition are not completely clear, but may involve increased expression of matrix metalloproteases (MMP), confused ketone body levels and inflammation.

MMP activity is often increased in diabetic patients, indicating that higher plasma MMP activity increased BBB permeability in diabetic condition via degradation of tight junction proteins. Animal study (Hawkins et al., 2007a) showed that increase of BBB 
permeability to sucrose in streptozotocin-treated rats was associated with the decreased level of tight junction proteins occludin and ZO-1 and the increase of MMP activity in plasma, which verified above deduction. Insulin treatment may attenuate the BBB hyperpermeability to sucrose.

In diabetic ketoacidosis, the relationship between ketone bodies and brain metabolism is well-documented (Roe et al., 1996). Two ketone bodies $\beta$-hydroxybutyrate and acetoacetate cross BBB via a monocarboxylic acid transport system. This transport was increased by elevated blood concentration of ketone bodies. $\beta$ hydroxybutyrate and acetoacetate increased BBB permeability and induced brain edema via increasing the production of vascular endothelial growth factor and vasoconstrictor endothelin-1 (Isales et al., 1999).

Inflammatory mechanisms underlying vascular pathology in DM are possibly common to the vasculature in the periphery and CNS. Formation of advanced glycation end products (AGEs) via glycation of blood proteins is a consequence of hyperglycemia, and it results in decreased kidney function and small vessels pathology. AGEs accumulation may induce vascular inflammation by the interactions between AGEs and AGE-specific receptors (RAGE) (Meerwaldt et al., 2009). AGEs activation of endothelial RAGE promotes upregulation of endothelial adhesion molecules including vascular cell adhesion molecule 1 (VCAM-1) and activates transcription factor nuclear factor- $\kappa \mathrm{B}(\mathrm{NF}-\kappa \mathrm{B})$. The former increases monocyte adhesiveness and vascular permeability while the latter regulates multiple proinflammatory and proatherosclerotic target genes in endothelial and vascular smooth muscle cells as well as in macrophages (Piga et al., 2007).

\section{ABC TRANSPORTERS AT BBB AND DIABETES P-GP AND DIABETES}

Diabetes is often associated with disorder of energy metabolism and increases of pro-inflammatory cytokines in the systemic circulation, which inferred that diabetes may alter expression and function of $\mathrm{ABC}$ transporters at $\mathrm{BBB}$, in turn, affect $\mathrm{BBB}$ permeability. We once reported that diabetes down-regulated P-GP expression at $\mathrm{BBB}$ of diabetic rats, leading to increase in brain distribution of vincristine and rhodamine (Liu et al., 2006). Seventy two hour exposure to serum of diabetic rats down-regulated function and protein levels of P-GP in primarily cultured rat brain microvessel endothelial cells (rBMECs), which may be reversed by adding of insulin (Liu et al., 2008a). We also found that the effects of diabetes on Abcb1 mRNA levels was dependent on both brain regions and $A b c b 1$ species in streptozotocin (STZ)-induced diabetic rats. Diabetes clearly down-regulated Abcbla mRNA levels in the cerebral cortex of diabetic rats, while up-regulated Abcbla mRNA levels in hippocampus. It was contrast to finding in Abcbla mRNA that the levels of Abcblb mRNA cerebral cortex of diabetic rats were increased rather than decreased. The Abcblb mRNA levels were not significantly affected by diabetes. The increased P-GP proteins were also observed in hippocampus of diabetic rats (Zhang et al., 2011). Similarly, expression of P-GP in brain striatum of type 2 diabetic mice was increased (Wu et al., 2009). In vivo study showed that insulin treatment not only restored the impaired function and expression of P-GP at BBB by diabetes, but also upregulated expression and function of P-GP at
BBB of normal rats (Liu et al., 2008a). In rBMECs, insulin dosedependently increased expression and function of P-GP, which may be abolished by insulin receptor antibody (Liu et al., 2009). Above results give a clue that low level of insulin in plasma is a main factor resulting in impairment of P-GP expression at BBB. Further investigation showed that insulin up-regulated $\mathrm{P}-\mathrm{GP}$ expression and function at $\mathrm{BBB}$ via activating $\mathrm{PKC} / \mathrm{NF}-$ $\kappa \mathrm{B}$ pathway (Liu et al., 2009). NO is also considered to regulate expression and function of P-GP, but our previous study on Caco2 cells showed that the effects of NO donors on P-GP function and expression were dependent on exposure time. Four hour exposure to NO donors impaired P-gp function and expression, whereas 24-h exposure stimulated P-gp function and expression (Duan et al., 2012). Other factors such as inflammatory cytokines and related molecules (von Wedel-Parlow et al., 2009; Poller et al., 2010) may be involved in expression and function of P-GP at BBB. Several studies have showed that cytokines exert dose-and time-dependent modulation of efflux transporters. In RBE4 cells, tumor necrosis factor-alpha (TNF- $\alpha$ ) upregulated P-GP expression, accompanied by decrease in cellular uptake of the P-GP substrate vinblastine (Yu et al., 2007). In isolated rat brain capillaries, TNF- $\alpha$ shows biphasic effects on the expression and activity of P-GP with low dose decreasing but longer exposure $(6 \mathrm{~h})$ increasing the expression and activity of P-GP. This is probably explained by differences in the signaling pathways activated (Bauer et al., 2007). Following 72-h exposure to TNF- $\alpha$, human endothelial cells showed an increase of P-GP expression, but P-GP activity was unaltered (Poller et al., 2010). In primary cultures of porcine brain capillary endothelial cells, P-GP protein expression was transiently increased after TNF- $\alpha$ addition within $6 \mathrm{~h}$ of incubation followed by a reduction after 24 and $48 \mathrm{~h}$, whereas the Abcb1 mRNA levels were not changed. It was also found that Interleukin-1 $\beta$ (IL-1 $\beta$ ) decreased the expression and activity of P-GP (von Wedel-Parlow et al., 2009).

Absence of functional P-GP at BBB leads to highly increased brain penetration of a number of important drugs, inducing dramatically increased neurotoxicity, or fundamentally altered pharmacological effects of the drugs on CNS. Phenobarbital, an antiepileptic and sedative drug, was shown to be a substrate for P-GP (Yang and Liu, 2008). The impaired function and expression of P-GP on BBB of diabetic mice resulted in increased brain distribution and pharmacological effect of phenobarbital (Liu et al., 2007a). Kamei et al. also reported that the systemic administration of second-generation H1-receptor antagonists (epinastine and cetirizine) showed CNS depressant effects in STZ-induced diabetic mice, accompanied by lower P-GP expression levels in the whole brain (Kamei et al., 2005).

\section{BCRP AND DIABETES}

$\mathrm{BCRP}$, another $\mathrm{ABC}$ transporter was reported to be downregulated at $\mathrm{BBB}$ of diabetic rats, accompanied by increases in brain distribution of prazosine and cimetidine (Liu et al., 2007b). Insulin treatment may attenuate the impairment of Bcrp expression and function induced by diabetes. Neither aminoguanidine (an inhibitor of advanced glycation end product) nor metformin treatment prevented the impairment of Bcrp function and expression in brain cortex of diabetic rats although aminoguanidine 
and metformin levels of advanced glycation end product and glucose in plasma diabetic rats were significantly suppressed by aminoguanidine and metformin respectively, indicating contribution of insulin to downregulation of BCRP. However, in rBMECs, we found that unlike P-GP, insulin itself suppressed rather than increased expression and function of Bcrp (Liu et al., 2011), indicating existence of other factors impairing expression and function of BCRP at BBB. von Wedel-Parlow et al. reported that TNF- $\alpha$ and IL- $1 \beta$ rapidly decreased Abcg 2 mRNA expression within $6 \mathrm{~h}$. Long-term treatment with the vasoconstrictor endothelin-1 (TNF- $\alpha$ downstream agent) induced Abcg2 protein expression. IL-1 $\beta$ caused a continuous decrease in protein expression of BCRP (von Wedel-Parlow et al., 2009). In immortalized brain endothelial cells (hCMEC/D3), IL-1 $\beta$, IL-6 and TNF- $\alpha$ significantly reduced levels of BCRP mRNA. IL- $1 \beta$ showed the strongest inhibitory effect on expression of BCRP protein among the three proinflammatory cytokines. BCRP activity was also significantly inhibited by IL- $1 \beta$, IL- 6 and TNF- $\alpha$, assessed by mitoxantrone uptake experiments (Poller et al., 2010).

\section{ABC TRANSPORTERS, DIABETES AND ALZHEIMER'S DISEASE $A B C$ transporters mediate transport of $A \beta$ across $B B B$}

Recent studies have revealed that DM is a risk factor for cognitive dysfunction or dementia, especially those related to $\mathrm{AD}$. $\mathrm{AD}$ is characterized by generation and deposition of amyloid $\beta$-peptide $(\mathrm{A} \beta)$ within extracellular spaces of the brain, which is believed to be the key player in the pathogenesis of AD (Vogelgesang et al., 2002). Animal and clinical reports showed that diabetes increased accumulation of $\mathrm{A} \beta$ in brain (Liu et al., 2008b; Alafuzoff et al., 2009; Tomita et al., 2013) although roles of $A \beta$ in $A D$ progression with diabetes need further verification. Possible relationships in diabetes, $\mathrm{BBB}$ function, $\mathrm{A} \beta$, and $\mathrm{AD}$ were shown in Figure 1.

Several studies (Kuhnke et al., 2007; Jedlitschky et al., 2010) have showed that P-GP (ABCB1) mediated transport of $A \beta$ across $\mathrm{BBB}$. Our previous study demonstrated that diabetes increased $\mathrm{A} \beta$ level in hippocampus and cortex of rats. Insulin treatment may restore the increase of $A \beta$ level induced by diabetes. Further study showed that the increase may partly come from both decrease in efflux of $A \beta$ from brain to blood and increase in influx of $A \beta$ from blood to brain, which were in line with impairment of P-GP function and expression (Liu et al., 2008b). This finding infers that increased accumulation of brain $A \beta$ induced by diabetes at least is attributed to impaired expression and function of P-GP at $\mathrm{BBB}$, which seems to explain that diabetic condition contributes to the pathogenesis of AD (Liu et al., 2008b). In a clinical trial with patients with mild to moderate $\mathrm{AD}$ using rifampicin, a potent inducer of P-gp, a reduced cognitive decline was observed after 12 months of treatment, presumably because the drug was able to improve the clearance of $A \beta$ from the brain via the enhancement of P-gp mediated transport (Loeb et al., 2004). It was interesting that $A \beta$ itself was reported to down-regulate expression of P-GP in brain and dosing of the animals with the PXR ligand pregnenolone-16 alpha-carbonitrile upregulated $\mathrm{P}$-gp expression and reduced cerebral $\beta$-amyloid deposition (Hartz et al., 2010), inferring that accumulation of $A \beta$ in turn further downregulated P-GP expression at BBB.

BCRP also mediate transport of endogenous substances across BBB. Several reports (Tai et al., 2009; Xiong et al., 2009; Do et al., 2012) have verified that $B C R P$ mediate transport of $A \beta$ across $\mathrm{BBB}$. The role of ABCG2 in efflux of $\mathrm{A} \beta$ across $\mathrm{BBB}$ was investigated in Abcg2-null mice after intravenous injection of labeled A $\beta$ (Xiong et al., 2009; Shen et al., 2010; Zhang et al., 2013). The results demonstrated that Abcg2-null mice significantly accumulated more $A \beta$ in their brains than wild-type mice. In situ brain perfusion technique, GF120918 (dual inhibitor of Abcb1 and Abcg2) strongly enhanced the uptake of $[3 \mathrm{H}] \mathrm{A} \beta$ 1-40 by the brains of Abcb1-deficient mice, but not by the brains of Abcb1/Abcg2-deficient mice (Do et al., 2012). This role was further confirmed by in vitro studies utilizing ABCG2 overexpressing cells and hCMEC/D3 (Tai et al., 2009; Xiong et al., 2009). Above results give a concept that BCRP and P-GP "team up and work together" in transporting chemotherapeutics at BBB may possibly be extended to their handling of $\mathrm{A} \beta$ (Wolf et al., 2012). Unlike P-GP, Xiong and colleagues reported that ABCG2 gene was significantly up-regulated in the brains of $\mathrm{AD}$ patients and AD animals (Xiong et al., 2009), however, this induction was not copied by in vitro study on hCMEC/D3 (Xiong et al., 2009; Kania et al., 2011). The up-regulation of ABCG2 in AD brain was considered to be an adaptive response to relieve oxidative stress and protect brain cells/tissue against ROS induced damage and

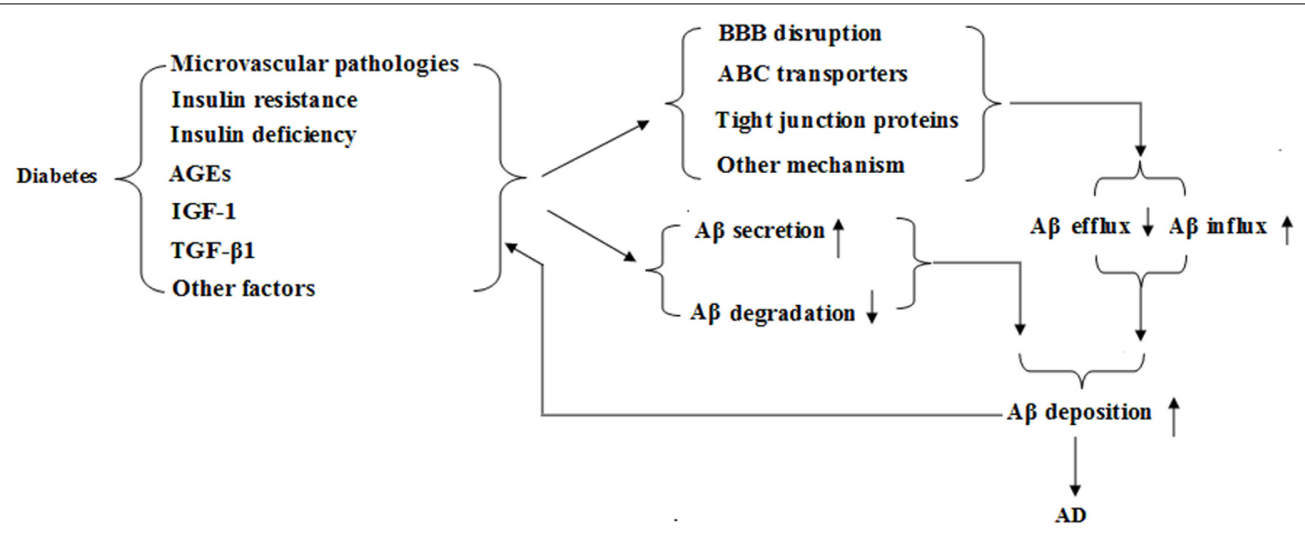

FIGURE 1 | Possible relationships in diabetes, blood brain barrier (BBB) function, $\beta$-amyloid and Alzheimer's disease (AD). 
inflammatory response via inhibiting NF- $\kappa \mathrm{B}$ signaling pathway (Shen et al., 2010). ABCG2 was also reported to plays a protective role against oxidative stress and anti-inflammation (Shen et al., 2010; Zeng et al., 2012). A study with HEK293 showed that transfection with ABCG2 significantly inhibited ROS formation by both tert-Butyl hydroperoxide and $\mathrm{H}_{2} \mathrm{O}_{2}$, indicating that ABCG2 decreased oxidative capacity of HEK293 cells or/and increased antioxidant capacity of the cells. Further investigation showed that over-expression of ABCG2 decreased $\mathrm{H}_{2} \mathrm{O}_{2}$-induced cell death, accompanied by decrease in IL- 8 secretion and inhibition of NF-kB activation. In the brains of Abcg2-knockout mice, the increased $A \beta$ accumulation was in line with NF- $\kappa B$ activation. In Neural-derived mouse neuroblastoma cell line 2a-695 cells (expresses APP and produces $\beta$-amyloid peptides), transfection with ABCG2 significantly decreased secretion of A $\beta 1-40$ peptides and downregulated activities of $\alpha-, \beta$, and $\gamma$ - secretases in cells (Shen et al., 2010). In Tg-SwDI/Abcg2-KO mice, Abcg2 deficiency increases oxidative stress and alters inflammatory response in the brain and exacerbates cognitive/memory deficit compared with Tg-SwDI mice, wild type mice and Abcg2-KO mice, accompanied by decrease in levels of total brain GSH and increase in levels of lipid/DNA oxidation (Zeng et al., 2012). All these results show important roles of BCRP in accumulation of $A \beta$ in brain of diabetic patients and animals.

$\mathrm{ABCB} 1$ and $\mathrm{ABCG} 2$ are not the only $\mathrm{ABC}$ transporters found to be associated with $A \beta$ transport. Two further $A B C$ transporters, $A B C A 7$ and $A B C C 1$, appeared on the stage of $A D$. In $A D$ patients ABCA7 expression was found to be higher than in non-demented people (Vasquez et al., 2013). Kim et al. evaluated the effect of ABCA7 on $A \beta$ pathology using ABCA7 knockout mice that were crossed to the J20 AD model. They found a robust increase of insoluble $A \beta$ and plaque load in the brains of ABCA7-deficient mice, but were unable to fully elucidate the mechanism behind this observation. Using bone marrow derived macrophages they showed a decreased ability of ABCA7 knockout cells to phagocytose oligomeric $A \beta$, pointing toward a possible mechanism of action (Kim et al., 2013). ABCC1 was also found to influence $A \beta$ brain burden. An up to 14-fold increase of $A \beta 42$ in ABCC1 knock-out mice is the so far greatest impact of an $\mathrm{ABC}$ transporter in AD mouse models. Moreover, amyloid burden was reduced by up to $80 \%$ in APPPS 1 mice via functional activation of ABCC1 (Krohn et al., 2011). ABCC1 is expressed in capillary endothelia of the blood-brain barrier, in neural stem and progenitor cells (Schumacher et al., 2012). Its function is regulated by mitochondria and influenced by mitochondrial polymorphisms (Scheffler et al., 2012). However, it is also different to the other transporters because one important site of high expression in the brain is the choroid plexus.

\section{Insulin/IGF-1 and TGF- $\beta 1$ are involved in A $\beta$ homeostasis}

Insulin resistance, which is often associated with type $2 \mathrm{DM}$, may induce a deficiency of insulin effects in the CNS. Some research suggests that insulin resistance accelerates Alzheimerrelated pathology through its effects on $A \beta$ metabolism. Recent data also suggest that brains of patients with $\mathrm{AD}$ are insulin and insulin-like growth factor-1 (IGF-1) resistant. $\beta$-amyloid oligomers are responsible for downregulation of neuronal insulin receptors, whereas $A \beta$ monomers are able to activate insulin/IGF1 receptor signaling. It has been hypothesized that depletion of $\beta$-amyloid monomers, occurring in the preclinical phase of $\mathrm{AD}$, might be the cause of early insulin/IGF-1 signaling disturbances that anticipate cognitive decline (Giuffrida et al., 2012).

Insulin directly increases $A \beta$ secretion from neurons by accelerating peptide trafficking to the plasma membrane (Gasparini et al., 2001) and promotes $A \beta$ degradation by regulating the expression of the insulin-degrading enzyme, a metalloprotease that catabolizes both insulin and A $\beta$ (Zhao et al., 2004). IGF1 increases $A \beta$ clearance from the brain by enhancing transport of $A \beta$-carrier proteins (e.g., albumin and transthyretin) into the brain (Carro et al., 2002). Hence, insulin and IGF-1 seem to act in conjunction as regulators of brain $A \beta$ content, and systemic conditions altering their interplay could indirectly promote $A \beta$ oligomerization. For example, aging (the main risk factor for $\mathrm{AD}$ ) is associated with low serum levels of IGF-1 (Piriz et al., 2011), and type 2 diabetes is associated with peripheral hyperinsulinemia and low brain insulin levels that could result in reduced $A \beta$ clearance (Giuffrida et al., 2012). Insulin and IGF-1 regulate multicargotransporters influencing trafficking of several molecules including $A \beta$ from the brain to the blood as well as to the cerebrospinal fluid (CSF) and possibly vice versa. Furthermore, insulin and related peptides regulate neurovascular coupling changing regional blood flow. Thus, positive effects of peripheral insulin/IGF-1 administration on AD pathology might be due to changes in the $\mathrm{BBB}$ and/or in the transport between the $\mathrm{CSF} /$ blood and the brain. Haploinsufficiency of the IGF-1 receptor (IGF-1R) (IGF-1R+/- mice) as well as neuronal deficiency of the insulin receptor (IR) (nIR-/- mice) or IGF-1R (nIGF-1R-/mice) leads to delayed $A \beta$ accumulation when crossed with mouse models for AD. Furthermore, insulin receptor substrate (IRS)2 knockout mice (IRS-2-/- mice) show reduced $A \beta$ levels in an Alzheimer background. These data suggest beneficial effects of decreased neuronal insulin/IGF-1 signaling on Alzheimerpathology and question the therapeutic outcome of long-term administration of insulin or IGF-1 in patients with AD (Zemva and Schubert, 2014).

TGF- $\beta 1$ is well-known to modulate blood vessel development and maturation. Oligodendrocyte precursor cells (OPCs) are an important source of TGF- $\beta 1$ that supports BBB integrity during development. OPCs derived TGF- $\beta 1$ activates the MEK/ERK pathway in cerebral endothelium via TGF- $\beta$ receptor, and the signaling cascade eventually increases the expression levels of tight-junction proteins to promote the BBB integrity (Seo et al., 2014 ). Reducing neuronal TGF- $\beta 1$ signaling in mice resulted in age-dependent neurodegeneration and promoted $A \beta$ accumulation and dendritic loss in a mouse model of AD (Wyss-Coray et al., 2001). In cultured cells, reduced TGF- $\beta$ signaling caused neuronal degeneration and resulted in increased levels of secreted $\mathrm{A} \beta$ and $\beta$-secretase-cleaved soluble amyloid precursor protein (Tesseur et al., 2006). Hence, reduced neuronal TGF- $\beta 1$ signaling increases age dependent neurodegeneration and $\mathrm{AD}$-like symptoms. Improvement of TGF- $\beta 1$ signaling may be a novel therapeutic approach to $\mathrm{AD}$, simultaneously targeting a neurodegenerative pathway and preventing $\mathrm{A} \beta$ deposition (Caraci et al., 2012). A recent study reported that TGF- $\beta 1$ potently regulates 
P-GP activity and ABCB1 mRNA at the developing BBB and both ALK5 and ALK1 pathways are implicated in the regulation of P-GP function (Baello et al., 2014). The research indicates that aberrations in TGF- $\beta 1$ levels at the BBB may lead to substantial changes in brain exposure to P-GP substrates including $A \beta$, triggering consequences for neurodegenerative disease. It is consistent with the above findings which illustrate that neuronal TGF- $\beta 1$ signaling has been impaired in $\mathrm{AD}$, resulting in impaired function and expression of P-GP at the BBB.

However, the regulation of BBB by TGF- $\beta 1$ is contradictory in DM. It is generally accepted advanced glycation endproducts (AGEs) are considered to be an important factor in the formation of diabetic vascular complications. Takeshita et al. reported that AGEs decreases in the expression of proteins comprising tight junctions through the production of TGF- $\beta 1$ in the BBB (Takeshita et al., 2014). This finding suggests high level of AGEs activate brain TGF- $\beta 1$ signal pathway, leading to disturbance of BBB under diabetic condition. Clinical and animal study also showed that elevated plasma and urinary levels of TGF- $\beta 1$ in diabetic patients and inhibition of TGF- $\beta 1$ action is beneficial for diabetic nephropathy (Li and Hölscher, 2007).

\section{FUTURE PERSPECTIVES}

$\mathrm{ABC}$ transporters including P-GP, BCRP and MRPs at BBB play an important role in the transporting compounds across BBB. The deliberate modulating ABC-transporters may mediate the permeability of their substrates including drugs and endogenous substances across BBB, affecting activity/toxicity of their substrates on CNS. Based on current knowledge, we propose that P-GP and BCRP are able to contribute to brain complications under diabetic conditions. However, we remain far from the exact contribution these transporters have to the disease. For example, how diabetes affect $A B C$ transporters in brain is still unclear. Thus, a better understanding of $\mathrm{ABC}$ transporters functions in the human brain is of major pharmacological importance to the development and optimization of therapeutic strategies that target these transporters.

Here, we only discussed alterations in expression and function of P-GP and BCRP at BBB under diabetic status. In fact, other transporters such as MRP2 (Hawkins et al., 2007b) and ABCA1 (Wang et al., 2012) were also investigated in brain of diabetic rats, but the clinical significances needs further investigation. In addition, it should be mentioned that effects of diabetes on expression and function of $\mathrm{ABC}$ transporters at $\mathrm{BBB}$ are often dependent on species of $A B C$ transporters, brain regions, duration of diabetes and diabetic type, which may gave different results.

\section{ACKNOWLEDGMENTS}

The project was supported by the National Science foundation of China (No. 81373482, No. 81473273 and No. 81102503) and the Fundamental Research Funds for the Central Universities (ZD2014YX0026, PT2014YX0057).

\section{REFERENCES}

Abbott, N. J., Patabendige, A. A., Dolman, D. E., Yusof, S. R., and Begley, D. J. (2010). Structure and function of the blood-brain barrier. Neurobiol. Dis. 37, 13-25. doi: 10.1016/j.nbd.2009.07.030
Alafuzoff, I., Aho, L., Helisalmi, S., Mannermaa, A., and Soininen, H. (2009). Beta-amyloid deposition in brains of subjects with diabetes. Neuropathol. Appl. Neurobiol. 35, 60-68. doi: 10.1111/j.1365-2990.2008.00948.x

Alves, M. G., Oliveira, P. F., Socorro, S., and Moreira, P. I. (2012). Impact of diabetes in blood-testis and blood-brain barriers: resemblances and differences. Curr. Diabetes. Rev. 8, 401-412. doi: 10.2174/157339912803529896

Baello, S., Iqbal, M., Bloise, E., Javam, M., Gibb, W., and Matthews, S. G. (2014). TGF- $\beta 1$ regulation of multidrug resistance P-glycoprotein in the developing male blood-brain barrier. Endocrinology 155, 475-484. doi: 10.1210/en. 2013-1472

Banks, W. A., Jaspan, J. B., and Kastin, A. J. (1997). Effect of diabetes mellitus on the permeability of the blood-brain barrier to insulin. Peptides 18, 1577-1584. doi: 10.1016/S0196-9781(97)00238-6

Bauer, B., Hartz, A. M., and Miller, D. S. (2007). Tumor necrosis factor alpha and endothelin-1 increase Pglycoprotein expression and transport activity at the blood-brain barrier. Mol. Pharmacol. 71, 667-675. doi: 10.1124/mol.106.029512

Caraci, F., Spampinato, S., Sortino, M. A., Bosco, P., Battaglia, G., Bruno, V., et al. (2012). Dysfunction of TGF- $\beta 1$ signaling in Alzheimer's disease: perspectives for neuroprotection. Cell. Tissue. Res. 347, 291-301. doi: 10.1007/s00441-0111230-6

Carro, E., Trejo, J. L., Gomez-Isla, T., LeRoith, D., and Torres-Aleman, I. (2002). Serum insulin-like growth factor I regulates brain amyloid-beta levels. Nat. Med. 8, 1390-1397. doi: 10.1038/nm1202-793

Chehade, J. M., Haas, M. J., and Mooradian, A. D. (2002). Diabetes-related changes in rat cerebral occludin and zonula occludens- 1 (ZO-1) expression. Neurochem. Res. 27, 249-252. doi: 10.1023/A:1014892706696

Cornford, E. M., Hyman, S., Cornford, M. E., and Clare-Salzler, M. (1995). Downregulation of blood-brain glucose transport in the hyperglycemic nonobese diabetic mouse. Neurochem. Res. 20, 869-873.

Dai, J. P., Vrensen, G. F. J. M., and Schlingemann, R. O. (2002). Blood-brain barrier integrity is unaltered in human brain cortex with diabetes mellitus. Brain Res. 954, 311-316. doi: 10.1016/S0006-8993(02)03294-8

De Vivo, D. C., Trifiletti, R. R., Jacobson, R. I., Ronen, G. M., Behmand, R., A., and Harik, S. I. (1991). Defective glucose transport across the blood-brain barrier as a cause of persistent hypoglycorrhachia, seizures, and developmental delay. N. Engl. J. Med. 325, 731-732.

Do, T. M., Noel-Hudson, M. S., Ribes, S., Besengez, C., Smirnova, M., Cisternino, S., et al. (2012). ABCG2- and ABCG4-mediated efflux of amyloid- $\beta$ peptide 1-40 at the mouse blood-brain barrier. J. Alzheimers Dis. 30, 155-166. doi: 10.3233/JAD-2012-112189

Duan, R., Hu, N., Liu, H. Y., Li, J., Guo, H. F., Liu, C., et al. (2012). Biphasic regulation of P-glycoprotein function and expression by NO donors in Caco-2 cells. Acta. Pharmacol. Sin. 33, 767-774. doi: 10.1038/aps.2012.25

Fanelli, C. G., Dence, C. S., Markham, J., Videen, T. O., Paramore, D. S., Cryer, P. E., et al. (1998). Blood-to-brain glucose transport and cerebral glucose metabolism are not reduced in poorly controlled type 1 diabetes. Diabetes 47, 1444-1450.

Gasparini, L., Gouras, G. K., Wang, R., Gross, R. S., Beal, M. F., Greengard, P., et al. (2001). Stimulation of beta-amyloid precursor protein trafficking by insulin reduces intraneuronal beta-amyloid andrequires mitogen-activated protein kinase signaling. J. Neurosci. 21, 2561-2570.

Giuffrida, M. L., Tomasello, F., Caraci, F., Chiechio, S., Nicoletti, F., and Copani, A. (2012). Beta-amyloid monomer and insulin/IGF-1 signaling in Alzheimer's disease. Mol. Neurobiol. 46, 605-613. doi: 10.1007/s12035-012-8313-6

Hartz, A. M., and Bauer, B. (2011). ABC transporters in the CNS - an inventory. Curr. Pharm. Biotechnol. 12, 656-673.

Hartz, A. M., Miller, D. S., and Bauer, B. (2010). Restoring blood-brain barrier P-glycoprotein reduces brain amyloid-beta in a mouse model of Alzheimer's disease. Mol. Pharmacol. 77, 715-723. doi: 10.1124/mol.109.061754

Hawkins, B. T., Lundeen, T. F., Norwood, K. M., Brooks, H. L., and Egleton, R. D. (2007a). Increased blood-brain barrier permeability and altered tight junctions in experimental diabetes in the rat: contribution of hyperglycaemia and matrix metalloproteinases. Diabetologia 50, 202-211. doi: 10.1007/s00125-006-0485-z

Hawkins, B. T., Ocheltree, S. M., Norwood, K. M., and Egleton, R. D. (2007b). Decreased blood-brain barrier permeability to fluorescein in streptozotocintreated rats. Neurosci. Lett. 411, 1-5. doi: 10.1016/j.neulet.2006.09.010

Hou, W. K., Xian, Y. X., Zhang, L., Lai, H., Hou, X. G., Xu, Y. X., et al. (2007). Influence of blood glucose on the expression of glucose transporter proteins 1 and 3 in the brain of diabetic rats. Chin. Med. J. (Engl). 120, 1704-1709. 
Hovsepyan, M. R., Haas, M. J., Boyajyan, A. S., Guevorkyan, A. A., Mamikonyan, A. A., Myers, S. E., et al. (2004). Astrocytic and neuronal biochemical markers in the sera of subjects with diabetes mellitus. Neurosci. Lett. 369, 224-227. doi: 10.1016/j.neulet.2004.07.071

Isales, C. M., Min, L., and Hoffman, W. H. (1999). Acetoacetate and betahydroxybutyrate differentially regulate endothelin-1 and vascular endothelial growth factor in mouse brain microvascular endothelial cells. J. Diabetes Complications 13, 91-97. doi: 10.1016/S1056-8727(99)00030-6

Jedlitschky, G., Vogelgesang, S., and Kroemer, H. K. (2010). MDR1- P-glycoprotein (ABCB1)-mediated disposition of amyloid- $\beta$ peptides: implications for the pathogenesis and therapy of Alzheimer's disease. Clin. Pharmacol. Ther. 88, 441-443. doi: 10.1038/clpt.2010.126

Kamei, J., Hirano, S., Miyata, S., Saito, A., and Onodera, K. (2005). Effects of first- and second-generation histamine-H1-receptor antagonists on the pentobarbital-induced loss of the righting reflex in streptozotocin-induced diabetic mice. J. Pharmacol. Sci. 97, 266-272. doi: 10.1254/jphs.FP00 40832

Kania, K. D., Wijesuriya, H. C., Hladky, S. B., and Barrand, M. A. (2011). Beta amyloid effects on expression of multidrug efflux transporters in brain endothelial cells. Brain Res. 1418, 1-11. doi: 10.1016/j.brainres.2011.08.044

Kim, W. S., Li, H., Ruberu, K., Chan, S., Elliott, D. A., Low, J. K., et al. (2013). Deletion of Abca7 increases cerebral amyloid- $\beta$ accumulation in the J20 mouse model of Alzheimer's disease. J. Neurosci. 33, 4387-4394. doi: 10.1523/JNEUROSCI.4165-12.2013

Krohn, M., Lange, C., Hofrichter, J., Scheffler, K., Stenzel, J., Steffen, J., et al. (2011). Cerebral amyloid- $\beta$ proteostasis is regulated by the membrane transport protein ABCC1 in mice. J. Clin. Invest. 121, 3924-3931. doi: 10.1172/JCI57867

Kuhnke, D., Jedlitschky, G., Grube, M., Krohn, M., Jucker, M., Mosyagin, I., et al. (2007). MDR1-P-Glycoprotein (ABCB1) Mediates Transport of Alzheimer's amyloid-beta peptides-implications for the mechanisms of Abeta clearance at the blood-brain barrier. Brain Pathol. 17, 347-753. doi: 10.1111/j.17503639.2007.00075.x

Li, L., and Hölscher, C. (2007). Common pathological processes in Alzheimer disease and type 2 diabetes: a review. Brain Res. Rev. 56, 384-402. doi: 10.1016/j.brainresrev.2007.09.001

Liu, H., Liu, X., Jia, L., Liu, Y., Yang, H., Wang, G., et al. (2008a). Insulin therapy restores impaired function and expression of P-glycoprotein in blood-brain barrier of experimental diabetes. Biochem. Pharmacol. 75, 1649-1658. doi: 10.1016/j.bcp.2008.01.004

Liu, H., Yang, H., Wang, D., Liu, Y., Liu, X., Li, Y., et al. (2009). Insulin regulates P-glycoprotein in rat brain microvessel endothelial cells via an insulin receptor-mediated PKC/NF-kappaB pathway but not a PI3K/Akt pathway. Eur. J. Pharmacol. 602, 277-282. doi: 10.1016/j.ejphar.2008.11.026

Liu, H. Y., Xu, X., Yang, Z. H., Deng, Y. X., and Liu, X. D. (2006). Impaired function and expression of P-glycoprotein in blood-brain barrier of streptozotocin-induced diabetic rats. Brain Res. 1123, 245-252. doi: 10.1016/j.brainres.2006.09.061

Liu, H., Zhang, D., Xu, X., Liu, X., Wang, G., Xie, L., et al. (2007a). Attenuated function and expression of P-glycoprotein at blood-brain barrier and increased brain distribution of phenobarbital in streptozotocin-induced diabetic mice. Eur. J. Pharmacol. 561, 226-232. doi: 10.1016/j.ejphar.2007.01.062

Liu, X., Jing, X. Y., Jin, S., Li, Y., Liu, L., Yu, Y. L., et al. (2011). Insulin suppresses the expression and function of breast cancer resistance protein in primary cultures of rat brain microvessel endothelial cells. Pharmacol. Rep. 63, 487-493. doi: 10.1016/S1734-1140(11)70515-1

Liu, Y. C., Liu, H. Y., Yang, H. W., Wen, T., Shang, Y., and Liu, X. D., et al. (2007b). Impaired expression and function of breast cancer resistance protein (Bcrp) in brain cortex of streptozocin-induced diabetic rats. Biochem. Pharmacol. 74, 1766-1772. doi: 10.1016/j.bcp.2007.08.021

Liu, Y., Liu, H., Yang, J., Liu, X., Lu, S., Wen, T., et al. (2008b). Increased amyloid betab-peptide (1-40) level in brain of streptozotocin-induced diabetic rats. Neuroscience 153, 796-802. doi: 10.1016/j.neuroscience.2008.03.019

Loeb, M. B., Molloy, D. W., Smieja, M., Standish, T., Goldsmith, C. H., Mahony, J., et al. (2004). A randomized, controlled trial of doxycycline and rifampin for patients with Alzheimer's disease. J. Am. Geriatr. Soc. 52, 381-387. doi: 10.1111/j.1532-5415.2004.52109.x

Mason, G. F., Petersen, K. F., Lebon, V., Rothman, D. L., and Shulman, G. I. (2006). Increased brain monocarboxylic acid transport and utilization in type 1 diabetes. Diabetes 55, 929-934. doi: 10.2337/diabetes.55.04.06.db05-1325
Meerwaldt, R., Zeebregts, C. J., Navis, G., Hillebrands, J. L., Lefrandt, J. D., and Smit, A. J. (2009). Accumulation of advanced glycation end products and chronic complications in ESRD treated by dialysis. Am. J. Kidney Dis. 53, 138-150. doi: 10.1053/j.ajkd.2008.08.031

Miller, D. S. (2014). ABC transporter regulation by signaling at the blood brain barrier: relevance to pharmacology. Adv. Pharmacol. 71, 1-24. doi: 10.1016/bs.apha.2014.06.008

Minamizono, A., Tomi, M., and Hosoya, K. (2006). Inhibition of dehydroascorbic acid transport across the rat blood-retinal and -brain barriers in experimental diabetes. Biol. Pharm. Bull. 29, 2148-2150. doi: 10.1248/bpb.29.2148

Obermeier, B., Daneman, R., and Ransohoff, R. M. (2013). Development, maintenance and disruption of the blood brain barrier. Nat. Med. 19, 1584-1596. doi: $10.1038 / \mathrm{nm} .3407$

Pahnke, J., Langer, O., and Krohn, M. (2014). Alzheimer's and ABC transportersnew opportunities for diagnostics and treatment. Neurobiol. Dis. S0969-9961, 00083-00087. doi: 10.1016/j.nbd.2014.04.001

Piga, R., Naito, Y., Kokura, S., Handa, O., and Yoshikawa, T. (2007) Short-term high glucose exposure induces monocyte-endothelial cells adhesion and transmigration by increasing VCAM-1 and MCP-1 expression in human aortic endothelial cells. Atherosclerosis 193, 328-334. doi: 10.1016/j.atherosclerosis.2006.09.016

Piriz, J., Muller, A., Trejo, J. L., and Torres-Aleman, I. (2011). IGF-I and the aging mammalian brain. Exp. Gerontol. 46, 96-99. doi: 10.1016/j.exger.2010.08.022

Poller, B., Drewe, J., Krähenbühl, S., Huwyler, J., and Gutmann, H. (2010). Regulation of BCRP (ABCG2) and P-glycoprotein (ABCB1) by cytokines in a model of the human blood-brain barrier. Cell. Mol. Neurobiol. 30, 63-70. doi: 10.1007/s10571-009-9431-1

Roe, T. F., Crawford, T. O., Huff, K. R., Costin, G., Kaufman, F. R., Nelson, M. D., et al. (1996). Brain infarction in children with diabetic ketoacidosis. J. Diabetes Complications 10, 100-108. doi: 10.1016/1056-8727(94)00058-1

Scheffler, K., Krohn, M., Dunkelmann, T., Stenzel, J., Miroux, B., Ibrahim, S. et al. (2012). Mitochondrial DNA polymorphisms specifically modify cerebral $\beta$-amyloid proteostasis. Acta Neuropathol. 124, 199-208. doi: 10.1007/s00401012-0980-x

Schumacher, T., Krohn, M., Hofrichter, J., Lange, C., Stenzel, J., Steffen, J., et al. (2012). ABC transporters B1, C1 and G2 differentially regulate neuroregeneration in mice. PLoS ONE 7:e35613. doi: 10.1371/journal.pone.0035613

Seo, J. H., Maki, T., Maeda, M., Miyamoto, N., Liang, A. C., Hayakawa, K., et al. (2014). Oligodendrocyte precursor cells support blood-brain barrier integrity via TGF- $\beta$ signaling. PLoS ONE 9:e103174. doi: 10.1371/journal.pone.0103174

Serlin, Y., Levy, J., and Shalev, H. (2011). Vascular pathology and blood-brain barrier disruption in cognitive and psychiatric complications of type 2 diabetes mellitus. Cardiovasc. Psychiatry Neurol. 2011:609202. doi: 10.1155/2011/609202

Shen, S., Callaghan, D., Juzwik, C., Xiong, H., Huang, P., and Zhang, W. (2010). ABCG2 reduces ROS-mediated toxicity and inflammation: a potential role in Alzheimer's disease. J. Neurochem. 114, 1590-1604. doi: 10.1111/j.14714159.2010.06887.x

Starr, J. M., Wardlaw, J., Ferguson, K., MacLullich, A., Deary, I. J., and Marshall, I. (2003). Increased blood-brain barrier permeability in type II diabetes demonstrated by gadolinium magnetic resonance imaging. J. Neurol. Neurosurg. Psychiatry 74, 70-76. doi: 10.1136/jnnp.74.1.70

Stumvoll, M., Goldstein, B. J., and van Haeften, T. W. (2005). Type 2 diabetes: principles of pathogenesis and therapy. Lancet. 365, 1333-1346.

Tai, L. M., Loughlin, A. J., Male, D. K., and Romero, I. A. (2009). P-glycoprotein and breast cancer resistance protein restrict apical-to-basolateral permeability of human brain endothelium to amyloid-beta. J. Cereb. Blood Flow. Metab. 29, 1079-1083. doi: 10.1038/jcbfm.2009.42

Takeshita, T., Nakagawa, S., Tatsumi, R., So, G., Hayashi, K., Tanaka, K., et al. (2014). Cilostazol attenuates ischemia-reperfusion-induced blood-brain barrier dysfunction enhanced by advanced glycation endproducts via transforming growth factor- $\beta 1$ signaling. Mol. Cell. Neurosci. 60, 1-9. doi: 10.1016/j.mcn.2014.01.006

Tesseur, I., Zou, K., Esposito, L., Bard, F., Berber, E., Can, J. V., et al. (2006). Deficiency in neuronal TGF-beta signaling promotes neurodegeneration and Alzheimer's pathology. J. Clin. Invest. 116, 3060-3069. doi: 10.1172/ JCI27341

Tomita, N., Furukawa, K., Okamura, N., Tashiro, M., Une, K., Furumoto, S., et al. (2013). Brain accumulation of amyloid $\beta$ protein visualized by positron emission tomography and BF-227 in Alzheimer's disease patients with or without 
diabetes mellitus. Geriatr. Gerontol. Int. 13, 215-221. doi: 10.1111/j.14470594.2012.00880.x

Vasquez, J. B., Fardo, D. W., and Estus, S. (2013). ABCA7 expression is associated with Alzheimer's disease polymorphism and disease status. Neurosci. Lett. 556, 58-62. doi: 10.1016/j.neulet.2013.09.058

Vogelgesang, S., Cascorbi, I., Schroeder, E., Pahnke, J., Kroemer, H. K., Siegmund, W., et al. (2002). Deposition of Alzheimer's $\beta$-amyloid is inversely correlated with P-glycoprotein expression in the brains of elderly nondemented humans. Pharmacogenetics 12, 535-541. doi: 10.1097/00008571-20021000000005

von Wedel-Parlow, M., Wölte, P., and Galla, H. J. (2009). Regulation of major efflux transporters under inflammatory conditions at the blood-brain barrier in vitro. J. Neurochem. 111, 111-118. doi: 10.1111/j.1471-4159.2009.06305.x

Wanek, T., Mairinger, S., and Langer, O. (2013). Radioligands targeting P-glycoprotein and other drug efflux proteins at the blood-brain barrier. J. Labelled Comp. Radiopharm. 56, 68-77. doi: 10.1002/jlcr.2993

Wang, X. T., Li, J., Liu, L., Hu, N., Jin, S., Liu, C., et al. (2012). Tissue cholesterol content alterations in streptozotocin-induced diabetic rats. Acta Pharmacol. Sin. 33, 909-917. doi: 10.1038/aps.2012.50

Wolf, A., Bauer, B., and Hartz, A. M. (2012). ABC transporters and the Alzheimer's disease enigma. Front. Psychiatry 3:54. doi: 10.3389/fpsyt.2012.00054

Wu, K. C., Pan, H. J., Yin, H. S., Chen, M. R., Lu, S. C., and Lin, C. J. (2009). Change in P-glycoprotein and caveolin protein expression in brain striatum capillaries in New Zealand obese mice with type 2 diabetes. Life Sci. 85, 775-781. doi: 10.1016/j.lfs.2009.10.014

Wyss-Coray, T., Lin, C., Yan, F., Yu, G. Q., Rohde, M., McConlogue, L., et al. (2001). TGF-betal promotes microglial amyloid-beta clearance and reduces plaque burden in transgenic mice. Nat. Med. 7, 612-618. doi: 10.1038/87945

Xiong, H., Callaghan, D., Jones, A., Bai, J., Rasquinha, I., Smith, C., et al. (2009). ABCG2 is upregulated in Alzheimer's brain with cerebral amyloid angiopathy and may act as a gatekeeper at the blood-brain barrier for Abeta (1-40) peptides. J. Neurosci. 29, 5463-5475. doi: 10.1523/JNEUROSCI.5103-08.2009

Yang, Z. H., and Liu, X. D. (2008). P-glycoprotein-mediated efflux of phenobarbital at the blood-brain barrier evidence from transport experiments in vitro. Epilepsy Res. 78, 40-49. doi: 10.1016/j.eplepsyres.2007.10.006

Yu, C., Pan, W., Tu, H., Waters, S., and Kastin, A. J. (2007). TNF activates MDR1 (P-glycoprotein) in cerebral microvascular endothelial cells. Cell. Physiol. Biochem. 20, 853-858. doi: 10.1159/000110445
Zemva, J., and Schubert, M. (2014). The role of neuronal insulin/insulin-like growth factor-1 signaling for the pathogenesis of Alzheimer's disease: possible therapeutic implications. CNS Neurol. Disord. Drug Targets 13, 322-337. doi: $10.2174 / 18715273113126660141$

Zeng, Y., Callaghan, D., Xiong, H., Yang, Z., Huang, P., and Zhang, W. (2012). Abcg2 deficiency augments oxidative stress and cognitive deficits in $\mathrm{Tg}$ SwDI transgenic mice. J. Neurochem. 122, 456-469. doi: 10.1111/j.14714159.2012.07783.x

Zhang, L. L., Lu, L., Jin, S., Jing, X. Y., Yao, D., Hu, N., et al. (2011). Tissue-specific alterations in expression and function of P-glycoprotein in streptozotocin-induced diabetic rats. Acta Pharmacol. Sin. 32, 956-966. doi: 10.1038/aps.2011.33

Zhang, W., Xiong, H., Callaghan, D., Liu, H., Jones, A., Pei, K., et al. (2013). Bloodbrain barrier transport of amyloid beta peptides in efflux pump knock-out animals evaluated by in vivo optical imaging. Fluids Barriers CNS. 10:13. doi: 10.1186/2045-8118-10-13

Zhao, L., Teter, B., Morihara, T., Lim, G. P., Ambegaokar, S. S., Ubeda, O. J., et al. (2004). Insulin-degrading enzyme as a downstream target of insulin receptor signaling cascade: implications forAlzheimer's disease intervention. J. Neurosci. 24, 11120-11126. doi: 10.1523/JNEUROSCI.2860-04.2004

Conflict of Interest Statement: The authors declare that the research was conducted in the absence of any commercial or financial relationships that could be construed as a potential conflict of interest.

Received: 23 August 2014; accepted: 24 November 2014; published online: 10 December 2014.

Citation: Liu L and Liu X-D (2014) Alterations in function and expression of ABC transporters at blood-brain barrier under diabetes and the clinical significances. Front. Pharmacol. 5:273. doi: 10.3389/fphar.2014.00273

This article was submitted to Experimental Pharmacology and Drug Discovery, a section of the journal Frontiers in Pharmacology.

Copyright (c) 2014 Liu and Liu. This is an open-access article distributed under the terms of the Creative Commons Attribution License (CC BY). The use, distribution or reproduction in other forums is permitted, provided the original author(s) or licensor are credited and that the original publication in this journal is cited, in accordance with accepted academic practice. No use, distribution or reproduction is permitted which does not comply with these terms. 